\title{
The Effect of People Movement on Wi-Fi Link Throughput in Indoor Propagation Environments
}

\author{
Nurul I. Sarkar \\ School of Computing and Mathematical Sciences \\ Auckland University of Technology \\ Auckland, New Zealand \\ nurul.sarkar@aut.ac.nz
}

\author{
Osman Mussa \\ School of Computing and Mathematical Sciences \\ Auckland University of Technology \\ Auckland, New Zealand \\ osmanmussa@gmail.com
}

\begin{abstract}
While various key performance limiting factors of IEEE 802.11-based Wi-Fi networks such as wireless protocols, radio propagation environment and signal interference have been studied by many network researchers, the effect of the movement of people (human) on Wi-Fi link throughput in indoor propagation environments has not been fully explored yet. This paper investigates the effect of people movement on Wi-Fi link throughput in six different indoor environments (i.e. lounge, bedroom, garage, common room, laboratory, and office space) using radio propagation measurements. Using a pair of wireless laptops we conducted various trials by considering both the straight line and random human movement in the above mentioned environments. Results obtained show that Wi-Fi link throughput degrades up to $20.4 \%$ as a result of people movement. The difference between the impact of straight line and random movement on Wi-Fi throughput is found to have insignificant. The research findings reported in this paper provide some insight into the impact of people movement on Wi-Fi link throughput in indoor environments.
\end{abstract}

Keywords: link throughput; people movement; radio propagation environments; Wi-Fi networks

\section{INTRODUCTION}

There has been a tremendous growth in the deployment of IEEE 802.11-based Wi-Fi networks in recent years. This growth is due to the flexibility, low cost, simplicity, and user mobility offered by the technology. This research aims to investigate the effect of people movement on Wi-Fi link throughput in six selected indoor propagation environments, such as lounge, bedroom, and garage in a suburban residential house and common room, laboratory, and obstructed office space in a multistory AUT Tower building. These indoor environments were selected because of the popularity of deploying Wi-Fi networks in these areas for accessing the Internet. This study is useful in the design, deployment, and capacity planning of indoor Wi-Fi systems.

A detailed discussion of wireless communications and radio propagation characteristics can be found in $[1,2]$, and Wi-Fi links and modules are discussed in the wireless networking literature [3, 4]. Many previous studies of indoor $\mathrm{Wi}-\mathrm{Fi}$ deployment have focused on improving the performance of Wi-Fi networks by quantifying the performance limiting-factors such as wireless network protocols, radio propagation environments and signal interference $[5,6]$.

In this paper we obtain some insight into the impact of people movement on Wi-Fi link throughput in the lounge, bedroom and garage in a suburban house, and common room, laboratory, and obstructed office space in the AUT Tower building.

Our approach is straightforward. Through a series of experiments/trials we study the effect of people movement on Wi-Fi link throughput in six selected indoor environments.

The remainder of this paper is organized as follows. Section II presents background and related work. The experiment details are described in Section III. The summary of research findings are presented in Section IV and a brief discussion in Section V concludes the paper.

\section{BACKGROUND AND RELATED WORK}

\section{A. Wi-Fi technology defined}

Wireless Fidelity (Wi-Fi) is a trade name for IEEE 802.1based wireless local area networks (WLANs). The 802.11 standard has been released in 1997. The specification requires a data transfer rate of from $1 \mathrm{Mbps}$ up to $2 \mathrm{Mbps}$ while retaining compatibility with existing LAN hardware and software infrastructure. The standard defines protocols for MAC layer and physical transmission in the unlicensed $2.4 \mathrm{GHz}$ radio band. After successful implementation by commercial companies such as Lucent Technologies, amendment was made for a better performance in the same year. The resulting standard was $802.11 \mathrm{~b}$, which specifies higher data transfer rates of 5.5 and $11 \mathrm{Mbps}$. The $802.11 \mathrm{~b}$ differs from the 802.11 in the MAC layer even though it retains compatibility with its predecessor. The physical layer is left unchanged. The $802.11 \mathrm{~b}$ standard was approved in 1999 and during that year the term Wi-Fi was introduced. The $802.11 \mathrm{~b}$ has proven to be very successful in the commercial domain. The majority of $\mathrm{Wi}-\mathrm{Fi}$ devices nowadays are made to the $802.11 \mathrm{~b}$ standard. 
In parallel with the $802.11 \mathrm{~b}$, another variant of the original 802.11 was also made. This variant is referred to as $802.11 \mathrm{a}$, which differs from both 802.11 and $802.11 \mathrm{~b}$ by using the $5 \mathrm{GHz}$ band rather than the $2.4 \mathrm{GHz}$ band. The 5 $\mathrm{GHz}$ radio band is unlicensed in the United States but not in many other countries especially in Europe. The 802.11a provides up to $54 \mathrm{Mbps}$, which is much faster than both 802.11 and $802.11 \mathrm{~b}$. However the use of different radio frequencies denies compatibility between 802.11a and $802.11 / 802.11 \mathrm{~b}$. Nevertheless the 802.11a was found satisfactory and approved in 1999.

The 802.11 standard consist of two important layers which is (1) MAC layer and; (2) Physical layer. Even though 802.11 take up different physical layer technologies, they still use the logical link control (LLC), media access control (MAC) and link layer address space (48bit). The first defined layer in an OSI model is the Physical layer (PHY), this layer is designed to carry raw bits of data rather than logical data packets over a physical link on connecting nodes. The second layer in the OSI model is the Data Link layer where it provided transfer of data between network entities.

\section{B. Related work}

There are a quite few attempts to study the performance of Wi-Fi networks in indoor radio propagation environments. In this paper, for brevity, we refer to only a selected set of literature on radio propagation measurements and modeling.

Punnoose et al. [7] conducted various experiments in a controlled laboratory environment to investigate the signal interference between Bluetooth and the $802.11 \mathrm{~b}$ direct sequence spread spectrum (DSSS) devices.

Schafer et al. [8] reported radio wave propagation measurements and simulation experiments in hospital settings. The attenuation of single walls and rooms was also investigated. A study was conducted by Messier et al. [9] at Worcester Polytechnic Institute to investigate the influence of various factors on the performance of Wi-Fi. One of the aims of the study was to find a correlation between received power and link throughput.

Sarkar and Sowerby [10] have investigated the performance of Wi-Fi link throughput in a crowded office environment. Using $802.11 \mathrm{~b}$ cards they examined the impact of line of sight (LOS) blockage by office walls and partitions on Wi-Fi link throughput.

Stein [11] discussed indoor WLAN performance where this was done in a dense office environment. During the experiments conducted, researchers found that when radio signals travelling from one node to another in an indoor environment, the waves reflect, scattered and diffracted against various objects.

The above review of literature reveals that the effect of the movement of people on Wi-Fi link throughput is an unexplored area in the field of Wi-Fi performance study. Radio propagation measurement approach was used for system performance evaluation. This measurement approach is exploratory in the sense that there was very limited prior research on the impact of people movement on Wi-Fi link throughput to guide this research endeavor. Therefore, an empirical study through propagation measurements was adopted in this study.

\section{EXPERIMENT DETAILS}

This section examines the effect of people movement (both straight line and random motion) on link throughput of a typical $802.11 \mathrm{~g}$ ad hoc network in the lounge, bedroom, garage in a suburban residential house; and common room, laboratory, and office space in the AUT Tower building. We first describe the measurement testbed and then present experiment details and results.

\section{A. Measurement testbed and resources used}

The experimental setup for investigating the impact of people movement consisted of a pair of $802.11 \mathrm{~g}$ laptops with identical configuration (Dell XPS15; MS Windows 7 Professional). The $802.11 \mathrm{~g}$ wireless USB adapters used in the experiment were NETGEAR (WNDA3100) high data rate (54 Mbps) (omnidirectional) with a power output of 14 $\mathrm{dBm}$ [12]. One of the laptops (i.e., USB card) was set as the transmitter (Tx) and the other as the receiver (Rx). Both the transmitting and receiving laptops were positioned at the height of the lap of a seated person during the experimentation by placing the laptops on chairs $(45 \mathrm{~cm}$ in height). For each experiment, a text file of size $129 \mathrm{MB}$ was sent from the Tx to the Rx using the 'File transmission' feature of the NETGEAR WNDA3100 Smart Wizard, which allows us to obtain the file transmission time [13]. The link throughput between the Tx and the Rx was computed by dividing the file size by the transmission time.

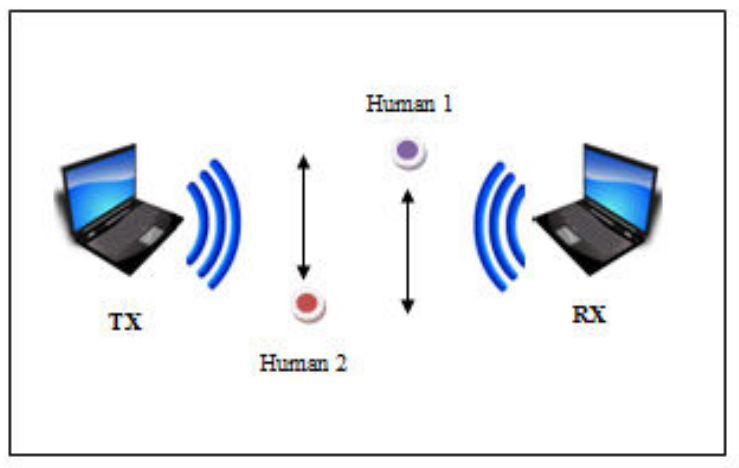

(a)

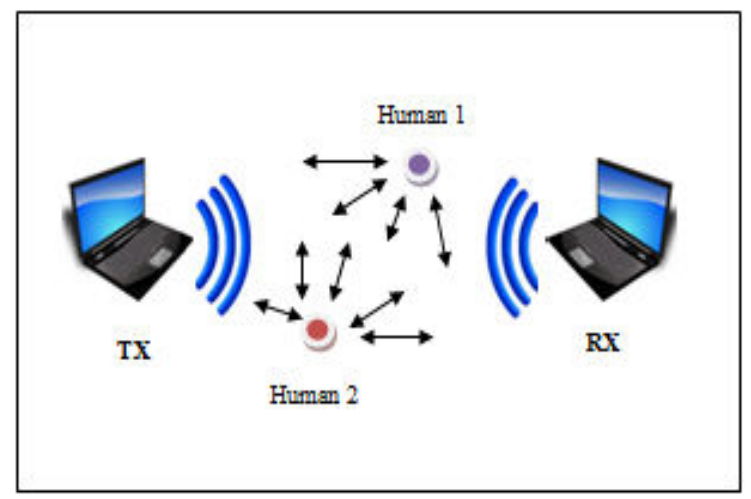

(b)

Figure 1. People movement in (a) Straight line; and (b) Random motion 
TABLE I. MEASUREMENT SCENARIOS AND EXPERIMENTS

\begin{tabular}{|c|c|}
\hline Scenarios & Experiment \\
\hline \begin{tabular}{ll}
\multicolumn{2}{l}{ Suburban house: } \\
1. & Lounge \\
2. & Bedroom \\
3. & Garage
\end{tabular} & $\begin{array}{l}\text { 1a. Effect of no human in the lounge } \\
\text { 1b. Effect of ST movement in the lounge } \\
\text { 1c. Effect of RM movement in the lounge } \\
\text { 2a. Effect of no human in the bedroom } \\
\text { 2b. Effect of ST movement in the bedroom } \\
\text { 2c. Effect of RM movement in the bedroom } \\
\text { 3a Effect of no human in the garage } \\
\text { 3b. Effect of ST movement in the garage } \\
\text { 3c. Effect of RM movement in the garage }\end{array}$ \\
\hline $\begin{array}{l}\text { 4. Common } \\
\text { room }\end{array}$ & $\begin{array}{l}\text { 4a. Effect of no human in the common room } \\
\text { 4b. Effect of ST movement in the common room } \\
\text { 4c. Effect of RM movement in the common room }\end{array}$ \\
\hline 5. Laboratory & $\begin{array}{l}\text { 5a. Effect of no human in the laboratory } \\
\text { 5b. Effect of ST movement in the laboratory } \\
\text { 5c. Effect of RM movement in the laboratory }\end{array}$ \\
\hline 6. Office space & $\begin{array}{l}\text { 6a. Effect of no human in the office space } \\
\text { 6b. Effect of ST movement in the office space } \\
\text { 6c. Effect of RM movement in the office space }\end{array}$ \\
\hline
\end{tabular}

\section{B. Measurment scenarios}

Table I lists the six propagation measurement scenarios (environments) and the corresponding experiments (total 18 experiments/trials) to study the effect of people movement on Wi-Fi link throughput. The six scenarios are lounge, bedroom, garage, common room, laboratory, and an obstructed office space. Figure 1 shows experimental setup for the investigation of people movements on Wi-Fi throughput. The straight line (SL) and random motion (RM) movements are shown in Fig. 1(a) and 1(b), respectively. Two human movements are considered throughout the experiments for each scenario. In case of SL movement, two people have continuously walked in SL facing each other between TX and RX. In case of RM, two people have continuously walked randomly during data transmission. However, the distance between two laptops was kept 2 meters throughout the experiment which provided good signal strength for data transmission between the TX and RX.

\section{Description of experiment}

Experiments 1 to 3 were conducted in a typical suburban residential house (single level) environment. The house is made up of plasterboard with external brick walls. The windows are made up glasses. The experimental results are summarized in Table II.

1) Experiment 1(a to c): Effect of people movement in the lounge

The effect of SL and RM movements on Wi-Fi (802.11g ad hoc network) link throughput in the lounge of a suburban residential home is investigated. Two laptops were placed approximately 2 meters away from the main door to get the good signal strength for transmission. To avoid the impact of other people movements and devices, the house was vacant and there were no other radio devices operating in the same frequency range throughout the experiments.

2) Experiment 2(a to c): Effect of people movement in the bedroom

The effect of SL and RM movements on Wi-Fi link throughput in the bedroom is investigated in this experiment. The laptops were placed between the bed and entrance door for good communication.

3) Experiment 3(a to c): Effect of people movement in the garage

The set up for this experiment is similar to Experiments 1 and 2 described above. The effect of SL and RM movements on Wi-Fi link throughput is investigated in this experiment. There was a car and other metal objects including shelves in the garage during experimentation.

Experiments 4 to 6 were conducted in a multistory office building (AUT Tower) in Auckland's Central Business District. The common room is located on the ground floor, the laboratory is on the $3^{\text {rd }}$ floor and the office space is on the $2^{\text {nd }}$ floor. Rooms are made up of concrete, the ceiling made of perforated particle boards for ventilation, and the windows are made of translucent glasses. As Experiments 1 to 3, the experimental results for the Experiments 4 to 6 are summarized in Table II.

\section{4) Experiment 4(a to c): Effect of people movement in} the common room

The effect of SL and RM movements on Wi-Fi link throughput in the common room is investigated. The common room had kitchen utensils, freeze, microwave, and wooden tables and chairs. The measurement laptops were placed near the entrance door for good communication.

\section{5) Experiment 5 (a to c): Effect of people movement in} the laboratory

In this experiment we investigated the effect of SL and RM movements on Wi-Fi link throughput in the laboratory where two laptops were placed near the back end of the lab for good communication. This laboratory had 30 PCs placed along each side of the room.

6) Experiment 6 (a to c): Effect of people movement in the office space

The effect of SL and RM movements on Wi-Fi link throughput in a typical staff office space is investigated. This office was full of papers, text books and items (very clutter). There were two wooden book shelves and a PC in the room. Two laptops were placed in the centre of the room for good communication.

\section{RESULTS AND DISCUSSION}

\section{A. Measurement results}

The experimental results obtained from propagation measurements are summarized in Table II. The six 
propagation environments/scenarios are shown in column 1. Column 2 shows Wi-Fi link throughput (in Mbps) without the effect of people movement. We consider this throughput as reference throughput in determining the throughput degradation as a result of people movement (see discussion below). The link throughputs for the effect of ST and RM movements are shown in column 3 and 5, respectively; and the corresponding throughput degradation are shown in column 4 and 6 , respectively.

By looking at the throughputs achieved without the effect of people movement, we observe that the maximum throughput achieved was $28 \mathrm{Mbps}$ in the lounge followed by bedroom (26.5 Mbps). The throughputs for the remaining four environments (garage, common room, lab, and office space) are slightly lower than the throughputs obtained both in the lounge and bedroom. This is mainly due to the nature of the propagation environments where measurements were carried out. For example, in the garage there was a car and other metal objects including garage door when measurements were taken resulting in lower throughput than the lounge and bedroom. Similarly, freeze and microwave in the common room, PCs and desks in the Lab, and book shelves containing books and other objects contributed to lower throughputs.

Now let us focus on the effect of SL and RM movements on Wi-Fi link throughputs. We defined a parameter called 'throughput degradation' to determine the amount of throughput degrades as a result of people movement with respect to throughput obtained without the effect of people movement (i.e. reference throughput).
The throughput degradation indicates the variation of link throughput with respect to the reference throughput. The movement of people (both SL and RM) in all six indoor propagation environments had dramatic effect on Wi-Fi link throughput. The human obstructions (in the form of people movement) reduces Wi-Fi link throughput significantly. For example, Wi-Fi $802.11 \mathrm{~g}$ link throughput degrades by 0.4 to $20 \%$ and 0.8 to $20.4 \%$ for SL and RM movements, respectively which is a significant performance drops.

The percentage difference of the throughput degradation of SL and RM movements is shown in Column 7. We observe that the movement of people in RM has slightly more impact on Wi-Fi link throughput than SL movement, but this throughput degradation is not very significant.

\section{B. Measurement accuracy and validation}

The accuracy of the measurement results was improved by addressing the following issues.

- People movement: The measurements were conducted after hours to avoid obstruction by other people/objects in the measurement locations.

- Co-channel interference: During propagation measurements, a quite a few neighbouring wireless networks were detected.

- Validation: Each experiment of the propagation measurement was repeated three times to obtain repeatability of results, ensuring the correctness of measured data collection.

TABLE II. EFFECT OF PEOPLE MOVEMENT ON WI-FI LINK THROUGHPUT.

\begin{tabular}{|c|c|c|c|c|c|c|}
\hline \multirow{3}{*}{$\begin{array}{l}\text { Propagation } \\
\text { Environment }\end{array}$} & \multirow{3}{*}{$\begin{array}{c}\text { Throughput } \\
\text { (in Mbps) } \\
\text { without } \\
\text { people } \\
\text { movement }\end{array}$} & \multicolumn{4}{|c|}{ Throughput with people movement } & \multirow{3}{*}{$\begin{array}{l}\text { Difference of } \\
\text { throughput } \\
\text { degradation of } \\
\text { SL and RM } \\
\text { movements } \\
(\%)\end{array}$} \\
\hline & & \multicolumn{2}{|c|}{ Straight line (SL) } & \multicolumn{2}{|c|}{ Random motion (RM) } & \\
\hline & & $\begin{array}{c}\text { Throughput } \\
\text { (Mbps) }\end{array}$ & $\begin{array}{c}\text { Throughput } \\
\text { degradation (\%) }\end{array}$ & $\begin{array}{c}\text { Throughput } \\
\text { (Mbps) }\end{array}$ & $\begin{array}{c}\text { Throughput } \\
\text { degradation (\%) }\end{array}$ & \\
\hline \multicolumn{7}{|c|}{ Suburban residential house } \\
\hline Lounge & 28.0 & 22.4 & 20.0 & 22.3 & 20.4 & 0.4 \\
\hline Bedroom & 26.5 & 23.8 & 10.2 & 23.2 & 12.5 & 2.3 \\
\hline Garage & 23.2 & 21.2 & 8.6 & 20.9 & 9.9 & 1.3 \\
\hline \multicolumn{7}{|l|}{ AUT Tower } \\
\hline Common room & 23.8 & 23.7 & 0.4 & 23.6 & 0.8 & 0.4 \\
\hline Laboratory & 21.5 & 20.5 & 4.7 & 20.4 & 5.1 & 0.4 \\
\hline Office space & 23.1 & 23 & 0.4 & 22.8 & 1.3 & 0.9 \\
\hline
\end{tabular}




\section{CONCLUSIONS}

This paper investigated the effect of the movement of people on Wi-Fi link throughput in the lounge, bedroom, garage, common room, laboratory, and an obstructed office space. Through a series of trials we gained an insight into the effect of both the straight line (SL) and random motion (RM) people movements on Wi-Fi link throughput. Results obtained have shown that $802.11 \mathrm{~g}$ Wi-Fi link throughput degrades up to $20.4 \%$ in the lounge as a result of the people movements in RM between $\mathrm{Tx}$ and $\mathrm{Rx}$. The line of sight blockage by human obstructions causing significant performance drops. We observed that Wi-Fi link throughput degradation varies with indoor propagation environments in which Wi-Fi networks operate. For example, the Wi-Fi 'throughput degradation' as a result of RM in the lounge, bedroom, garage, common room, laboratory, and office space are $20.4 \%, 12.5 \%, 9.9 \%, 0.8 \%$, $5.1 \%$, and $1.3 \%$, respectively. However, the difference of WiFi throughput degradation (in \%) between the effect of SL and $\mathrm{RM}$ movements is found to have insignificant. Therefore, the style of people movement (e.g. straight line, random motion) has very little impact as far as Wi-Fi throughput degradation is concerned.

An investigation of the influence of the movement of people on link throughput in a typical 802.11 network in outdoor environment is planned as an extension of the study reported here.

\section{REFERENCES}

[1] N. I. Sarkar and E. Lo, "Performance studies of $802.11 \mathrm{~g}$ for various AP configuration and placement," presented at the 2011 IEEE Symposium on Computers and Informatics (ISCI 2011), Kuala Lumpur, Malaysia, March 20 - 22, 2011, pp. 29-34.

[2] V. K. Sakarellos, D. Skraparlis, A. D. Panagopoulos, and J. D. Kanellopoulos, "Optimum placement of radio relays in millimeter-wave wireless dual-hop networks," IEEE Antennas and Propagation Magazine, vol. 51, no. 2, pp. 190-199, 2009.

[3] M. Abolins, "Boost your Wi-Fi signal," in PC Upgrade, 2004, pp. 54-57.

[4] S. Swan, "WiFi 21st Century Cat's Whiskers," in Silicon Chip, 2002, pp. 66-70.

[5] L. Dong, "Opportunistic media access control and routing for delaytolerant mobile ad hoc networks," Wireless Networks, vol. 18, no. 8, pp. 949-965, 2012.

[6] N. I. Sarkar, "Improving WLAN performance by modifying an IEEE 802.11 MAC protocol," International Journal of Wireless Networks and Broadband Technologies (IJWNBT), vol. 1, no. 1, pp. 15-31, 2011.

[7] R. J. Punnoose, R. S. Tseng, and D. D. Stancil, "Experimental results for interference between Bluetooth and IEEE 802.11b DSSS systems," presented at the 54th IEEE Vehicular Technology Conference (VTC'01Fall), October 7-11, 2001, pp. 67-71.

[8] T. M. Schafer, J. Maurer, and W. Wiesbeck, "Measurement and simulation of radio wave propagation in hospitals," presented at IEEE 56th Vehicular Technology Conference (VTC '02-Fall), September 2428, 2002, pp. 792-796.

[9] A. Messier, J. Robinson, and K. Pahlavan, " Performance monitoring of a wireless campus area network," presented at the 22nd Annual Conference on Local Computer Networks, November 2-5, 1997, pp. 232-238.

[10] N. I. Sarkar and K. W. Sowerby, "Wi-Fi performance measurements in the crowded office environment: a case study," presented at the 10th IEEE International Conference on Communication Technology (ICCT ' 06), Guilin, China, November 27-30, 2006, pp. 37-40.

[11] J. C. Stein, "Indoor radio WLAN performance part II: Range performance in a dense office environment," Intersil Corporation 1997.

[12] "User Manual for the NETGEAR Wireless USB 2.0 Adapter," Santa Clara, USA, February 2004.

[13] NETGEAR WND A3100. Retrieved January 20, 2013, from http://www.netgear.com/ 